R. E. Svetic, Department of Mathematics, Michigan State University, East Lansing, MI 48824-1027. e-mail: rsvetic@nemo.mth.msu.edu

H. Volkmer, Department of Mathematical Sciences, University of Wisconsin

- Milwaukee, P. O. Box 413, Milwaukee, WI 53201

\title{
THE MINIMAL DENJOY INDEX OF A SYMMETRIC PERFECT SET
}

\begin{abstract}
It is shown that every uniform symmetric perfect set admits a minimal Denjoy Index. This minimal Denjoy Index and a point where it is attained is found for many uniform symmetric perfect sets.
\end{abstract}

\section{Introduction}

The Denjoy Index $\alpha(x)$ of a point $x$ in a perfect set $P$ of real numbers measures the size of complementary intervals relative to $x$. It is equal to $(1-\operatorname{sp}(x))^{-1}$, where $\operatorname{sp}(x)$ is the symmetric porosity of $x$; see [1]. Denjoy used the Index extensively in his books [2]. He also applied the Index to symmetric perfect sets (Cantor-like sets) but did not carry out a detailed investigation. Recently, Evans, Humke and Saxe [3] and the authors [4] found several new results on the Denjoy Index of symmetric perfect sets. Before we explain some of these results, let us briefly review the simple definitions of the Denjoy Index and symmetric perfect sets.

Consider a perfect set $P$ with $x \in P$. Let $(a, b)$ and $(c, d)$ be any complementary intervals of $P$ such that $a<b \leq x \leq c<d$. If we reflect $(a, b)$ at $x$, we obtain the interval $(2 x-b, 2 x-a)$. Consider the intersection $I$ of this interval with $(c, d)$. If $I$ is nonempty, write $I=(x+s, x+t)$. Thus

$$
s=\max \{x-b, c-x\} \text { and } t=\min \{x-a, d-x\} .
$$

Then the Denjoy Index $\alpha(x)$ of $x$ is given by

$$
\alpha(x):=\lim _{h \rightarrow 0} \sup \left\{\frac{t}{s} \mid t<h\right\},
$$

\footnotetext{
Key Words: Denjoy Index, Symmetric Porosity, Symmetric Perfect Set

Mathematical Reviews subject classification: Primary: 26A03; Secondary: 04A15, 28A05, 54H05

Received by the editors December 29, 1998
} 
where the supremum is taken over all quotients $t / s$ with $t<h$ obtained in the way described above (the supremum of the empty set is defined as 1.)

Let $\lambda_{n}, n \in \mathbb{N}:=\{1,2,3, \ldots\}$, be a sequence of positive numbers such that

$$
\sum_{m=1}^{\infty} \lambda_{m}=1 \quad \text { and } \quad \mu_{n}:=\sum_{m=n+1}^{\infty} \lambda_{m}<\lambda_{n}
$$

for all $n \in \mathbb{N}$ (for convenience define $\mu_{0}=1$ ). Then the set

$$
P:=\{T(X) \mid X \subset \mathbb{N}\}
$$

where $T(X):=\sum_{n \in X} \lambda_{n}$, is a symmetric perfect set. A convenient way to refer to an arbitrary point $x=T(X) \in P$ is to identify the subset $X$ of $\mathbb{N}$ with the 01-sequence $x_{n}, n \in \mathbb{N}$, where $x_{n}=0$ or $x_{n}=1$ according to whether $n \notin X$ or $n \in X$. This identification defines a one-to-one correspondence between points in $P$ and 01-sequences.

That our definition of symmetric perfect sets agrees with the one given in [3] can be seen as follows (note that $\alpha_{n}$ in [3] is identical to our $\epsilon_{n}$ ). Define $\eta_{n}:=\mu_{n} / \lambda_{n} \in(0,1)$ and $\epsilon_{n}:=\left(1-\eta_{n}\right) /\left(1+\eta_{n}\right) \in(0,1)$. Observe that $P$ is obtained in a similar way as the Cantor set by first removing the middle open interval $\left(\mu_{1}, \lambda_{1}\right)$ of length $\epsilon_{1}$ from $[0,1]$, then removing open intervals $\left(\mu_{2}, \lambda_{2}\right)$ and $\left(\lambda_{1}+\mu_{2}, \lambda_{1}+\lambda_{2}\right)$ of fractional length $\epsilon_{2}$ from the remaining two closed intervals and so on, showing that $P$ is a symmetric perfect set.

If $\eta_{n}=\eta$ is constant, then we say that $P=P(\eta)$ is a uniform symmetric perfect set and $\mu_{n}=\frac{\eta^{n}}{(1+\eta)^{n}}$ and $\lambda_{n}=\frac{\eta^{n-1}}{(1+\eta)^{n}}$.

The Denjoy Index defines a function $\alpha: P(\eta) \rightarrow[1, \infty)$. The maximum value of this function is $1-\eta+\frac{1}{\eta}$ as shown in [3]. In [4] it is proved that the minimal value of $\alpha$ is $\frac{1+\eta-\eta^{2}}{\eta(1+\eta)}$ if $0<\eta \leq(\sqrt{5}-1) / 2$, and it is attained at the point given by the two-periodic sequence $101010 \ldots$. This leaves open the question of the minimal Denjoy Index of $P(\eta)$ if $(\sqrt{5}-1) / 2<\eta<1$.

The new results of this paper are the following. In Section 2 we prove that a minimal value of $\alpha$ on $P(\eta)$ exists. This is not clear from the definition because $\alpha$ is not a continuous function. We conjecture that the minimum is always attained at a periodic point (a point given by a periodic 01-sequence.)

In Section 3 we prove the Intersection Theorem 3.4 for complementary intervals of $P(\eta)$ which allows us to obtain a simpler expression for the Denjoy Index in Theorem 4.1 of Section 4. In particular, the Denjoy Index of a periodic point becomes accessible to computer calculations. At the end of Section 4 we present a computer generated table of those points that minimize the Denjoy Index on $P(\eta)$ among all periodic points of period at most 11 . 
The results from [4] and the table indicate that the point $w$ given by the two-periodic sequence $101010 \ldots$ is of special interest. In Section 5 we use the results from Section 4 to calculate the Denjoy Index of $w$. We find that it is a piecewise rational function of $\eta$ (with countably infinitely many "pieces"). In Theorem 5.3, we show that $w$ minimizes the Denjoy Index on $P(\eta)$ if $\eta$ belongs to a union of countably many disjoint intervals which accumulate at 1 .

An inspection of the table in Section 4 suggests several obvious conjectures that we leave for further investigation.

\section{Existence of the Minimal Denjoy Index}

Consider the uniform symmetric perfect set $P=P(\eta)$ for a given $\eta \in(0,1)$. The right endpoint of a bounded complementary interval of $P$ is given by $T(A)$ where $A$ is a finite nonempty subset of $\mathbb{N}$. We call $p:=\max A$ the level of the complementary interval. Its length is $\lambda_{p}-\mu_{p}$. The set $P$ has also two unbounded complementary intervals, namely $(-\infty, 0)$ and $(1, \infty)$. In all what follows, we will replace these intervals by the bounded intervals $(1-1 / \eta, 0)$ and $(1,1 / \eta)$ which we call complementary intervals of level 0 . Of course, this convention does not affect the definition of the Denjoy Index.

We define an auxiliary function $\beta: P \rightarrow[1, \infty]$ by

$$
\beta(x):=\sup \left\{\begin{array}{c|c}
\frac{t}{s} & \begin{array}{c}
a<b \leq x \leq c<d, \text { and }(a, b) \text { and }(c, d) \\
\text { are complementary intervals of } \mathrm{P}
\end{array}
\end{array}\right\},
$$

where $s, t$ are defined in (1.1). It is clear that

$$
\alpha(x) \leq \beta(x) \quad \text { for all } x \in P .
$$

We define a function $S: P \rightarrow P$ by

$$
S x:= \begin{cases}\frac{1+\eta}{\eta} x & \text { if } x<1 / 2 \\ \frac{1+\eta}{\eta} x-\frac{1}{\eta} & \text { if } x>1 / 2 .\end{cases}
$$

In terms of the 01-sequences representing points in $P$, the map $S$ is just the shift to the left in the space of 01-sequences.

Lemma 2.1. For every $x \in P$, we have that $\beta(x) \geq \beta(S x)$ and $\alpha(x)=\alpha(S x)$.

Proof. We assume that $x<1 / 2$ (the case $x>1 / 2$ is similar.) The function $x \mapsto \frac{1+\eta}{\eta} x$ maps $P \cap\left[0, \mu_{1}\right]$ bijectively onto $P$. The interval $\left(\mu_{1}, \lambda_{1}\right)$ is mapped onto $(1,1 / \eta)$, and the interval $\left(\mu_{1}-\lambda_{1}, 0\right)$ is mapped onto $(1-1 / \eta, 0)$. 
Therefore, $\beta(S x)$ equals the right-hand side of (2.1) but with the additional condition $c<1 / 2$ and $(a, b)=(1-1 / \eta, 0)$ replaced by the shorter interval $(a, b)=\left(\mu_{1}-\lambda_{1}, 0\right)$. Evidently, this implies $\beta(S x) \leq \beta(x)$. The second statement is proved in a similar way.

Lemma 2.2. For every $x \in P$, the sequence $\beta\left(S^{n} x\right)$ is nonincreasing and converges to $\alpha(x)$ as $n \rightarrow \infty$.

Proof. It follows from Lemma 2.1 that the sequence $\beta\left(S^{n} x\right)$ is nonincreasing. Moreover, by (2.2) and Lemma 2.1,

$$
\beta\left(S^{n} x\right) \geq \alpha\left(S^{n} x\right)=\alpha(x)
$$

for all $n \in \mathbb{N}$. Let $\epsilon>0$ be given. According to (1.2) choose $\delta>0$ such that

$$
\frac{t}{s} \leq \alpha(x)+\epsilon \text { if } t<\delta .
$$

Choose $m \in \mathbb{N}$ so large that $\lambda_{m}<\delta$. Remove from [0,1] all complementary intervals of level at most $m$, and let $J$ be the component of the resulting set containing $x$. Note that $J$ has length $\mu_{m}$. Let $I_{0}, I_{1}$ be the open intervals flanking $J$ of length $\lambda_{m}-\mu_{m}$. These intervals are contained in complementary intervals of $P$. By choice of $m, I_{0}, I_{1}$ and $J$ are all contained in $(x-\delta, x+\delta)$. As in the proof of the previous lemma, we see that $\beta\left(S^{m} x\right)$ equals the righthand side of (2.1) but with the additional constraint that $(a, b)$ and $(c, d)$ lie in $J$ or equal $I_{0}$ and $I_{1}$, respectively. Hence, by $(2.3), \beta\left(S^{m} x\right) \leq \alpha(x)+\epsilon$. Since $\epsilon>0$ was arbitrary, this yields that $\beta\left(S^{n} x\right)$ converges to $\alpha(x)$.

For $n \in \mathbb{N}$, we define $\beta_{n}(x)$ as $\beta(x)$ but allow only complementary intervals of level at most $n$. Note that $\beta_{n}$ is well-defined on the set $P_{n}$ that we obtain by removing all complementary intervals of level at most $n$ from $[0,1]$. For $x \in P$, we have that

$$
\beta_{1}(x) \leq \beta_{2}(x) \leq \cdots \leq \sup _{n \in \mathbb{N}} \beta_{n}(x)=\beta(x) .
$$

We now prove that $\alpha$ has a minimum value.

Theorem 2.3. The function $\beta$ is lower semicontinuous on $P$. There is $x \in P$ such that $\beta(x)=\min \beta(P)=\alpha(x)=\min \alpha(P)$.

Proof. Each function $\beta_{n}$ is continuous on $P$. It follows from (2.4) that $\beta$ is lower semicontinuous on $P$. Since $P$ is compact, there is $x \in P$ such that $\beta(x)=\min \beta(P)$. By Lemma 2.2 , the sequence $\beta\left(S^{n} x\right)$ is nonincreasing and converges to $\alpha(x)$. Since $\beta(x)$ is the minimum of $\beta(P)$, we obtain that $\beta(x)=$ $\beta(S x)=\cdots=\alpha(x)$. Finally, Lemma 2.2 implies that inf $\alpha(P)=\inf \beta(P)$. 


\section{The Intersection Theorem}

Let $P$ be a symmetric perfect set (not necessarily uniform). The complementary intervals of $P$ within $[0,1]$ have the form $\left(T(B)-\lambda_{p}+\mu_{p}, T(B)\right)$, where $B$ is a finite nonempty subset of $\mathbb{N}$ and $p=\max B$. We set $L(B)=T(B)-\lambda_{p}+\mu_{p}$. In the following we will always assume that the length $\lambda_{p}-\mu_{p}$ of complementary intervals of level $p$ is a nonincreasing function of $p$, that is, we assume that

$$
\lambda_{p}-\mu_{p} \geq \lambda_{p+1}-\mu_{p+1} \quad \text { for all } p \in \mathbb{N} .
$$

This condition is equivalent to the condition that

$$
\lambda_{p} \geq 2 \lambda_{p+1} \quad \text { for all } p \in \mathbb{N} .
$$

It is satisfied if $P$ is a uniform symmetric perfect set.

Let $A, B$ be two finite nonempty subsets of $\mathbb{N}$ such that

$$
-\frac{1}{2}<\sum_{i \in B} 2^{-i}-\sum_{i \in A} 2^{-i}<\frac{1}{2}
$$

Then there is a uniquely determined finite nonempty subset $C=F(A, B)$ of $\mathbb{N}$ such that

$$
\sum_{i \in B} 2^{-i}-\sum_{i \in A} 2^{-i}+\sum_{i \in C} 2^{-i}=\frac{1}{2}
$$

As an example, consider $A=\{3,5\}$ and $B=\{2,4\}$. Then $C=\{2,4,5\}$.

Note that $\max C \leq \max A \cup B$. Thus, by (3.1),

$$
T(C)-L(C) \geq \min \{T(A)-L(A), T(B)-L(B)\} .
$$

Lemma 3.1. Let $\lambda_{n}, n \in \mathbb{N}$, be any sequence of positive numbers which satisfies condition (3.2). Let $c_{1}, \ldots, c_{k}$ be integers with $c_{n} \geq-1$ for all $n=$ $1, \ldots, k$. Then

$$
\sum_{n=1}^{k} c_{n} 2^{-n}=0 \text { implies } \sum_{n=1}^{k} c_{n} \lambda_{n} \leq 0 .
$$

Proof. The proof is by induction on $k$. The case $k=1$ is trivial. Assume that the lemma is true with $k$ replaced by $k-1$ and let $c_{1}, \ldots, c_{k} \geq-1$ be integers such that $\sum_{n=1}^{k} c_{n} 2^{-n}=0$. Then $c_{k}$ must be even and since $c_{k} \geq-1$ we have that $c_{k} \geq 0$. Since the case $c_{k}=0$ is trivial, we can write $c_{k}=2 m$ 
with $m$ a positive integer. Using assumption (3.2), we obtain

$$
\begin{aligned}
\sum_{n=1}^{k} c_{n} \lambda_{n} & =\sum_{n=1}^{k-2} c_{n} \lambda_{n}+c_{k-1} \lambda_{k-1}+2 m \lambda_{k} \\
& \leq \sum_{n=1}^{k-2} c_{n} \lambda_{n}+\left(c_{k-1}+m\right) \lambda_{k-1} \leq 0
\end{aligned}
$$

where we used the induction hypothesis at the end.

Lemma 3.2. Let $A, B$ be two finite nonempty subsets of $\mathbb{N}$ which satisfy condition (3.3). Let $C=F(A, B)$ be determined according to (3.4). Then the following inequalities hold

$$
\begin{aligned}
& T(B)-T(A)+T(C) \leq \lambda_{1}, \\
& T(B)-T(A)+L(C) \geq \mu_{1}, \\
& L(B)-L(A)+L(C) \geq \mu_{1}, \\
& L(B)-L(A)+T(C) \leq \lambda_{1} .
\end{aligned}
$$

Proof. To prove the first inequality, write

$$
T(B)-T(A)+T(C)-\lambda_{1}=\sum_{i=1}^{k} c_{i} \lambda_{i},
$$

by collecting terms with the same $\lambda_{i}$. Clearly $c_{i} \in\{-1,0,1,2\}$ for $i \geq 2$. Note that also $c_{1} \geq-1$ because (3.4) implies that if $1 \in A$ then $1 \in B \cup C$. By definition of $C, \sum_{i=1}^{k} c_{i} 2^{-i}=0$. The first inequality now follows from Lemma 3.1.

If $A$ is a finite nonempty subset of $\mathbb{N}$, let $\tilde{A}$ be the finite nonempty subset of $\mathbb{N}$ determined by

$$
\sum_{i \in A} 2^{-i}+\sum_{i \in \tilde{A}} 2^{-i}=1
$$

Note that $C=F(A, B)$ implies $\tilde{C}=F(B, A)=F(\tilde{A}, \tilde{B})$. Also note that $L(A)=1-T(\tilde{A})$. We now obtain the second inequality from the first with $A$, $B$ interchanged and $C$ replaced by $\tilde{C}$.

The third inequality follows from the first with $A, B, C$ replaced by $\tilde{A}$, $\tilde{B}, \tilde{C}$, respectively. In a similar way, the fourth inequality follows from the second. 
We can now make a statement about the lengths of intersections of complementary intervals of $P$ and complementary intervals of a second shifted copy $y+P$ of $P$.

Lemma 3.3. Let $A, B$ be nonempty finite subsets of $\mathbb{N}$ satisfying (3.3), and let $C$ be defined according to (3.4). Let $y \in \mathbb{R}$ and set

$$
\begin{aligned}
& I:=(y+L(A), y+T(A)) \cap(L(B), T(B)), \\
& J:=(y+L(C), y+T(C)) \cap\left(\mu_{1}, \lambda_{1}\right) .
\end{aligned}
$$

Then $|I| \leq|J|(|I|$ denotes the length of the interval $I$.)

Proof. Let $p=\max A$ and $q=\max B$. We assume that $p \leq q$ (the case $p>q$ is treated similarly.) By (3.1),

$$
T(B)-L(B) \leq T(A)-L(A) .
$$

By (3.5),

$$
T(C)-L(C) \geq T(B)-L(B) .
$$

By (3.6) and since we may assume that $I$ is nonempty, there are three cases to consider.

Case 1: $y+L(A) \leq L(B)<T(B) \leq y+T(A)$.

Then, by Lemma 3.2 ,

$$
\begin{aligned}
& y+T(C) \leq L(B)-L(A)+T(C) \leq \lambda_{1}, \\
& y+L(C) \geq T(B)-T(A)+L(C) \geq \mu_{1} .
\end{aligned}
$$

Hence, by (3.7), $|J|=T(C)-L(C) \geq T(B)-L(B)=|I|$.

Case 2: $L(B) \leq y+L(A) \leq T(B) \leq y+T(A)$.

By Lemma 3.2, $y+L(C) \geq \mu_{1}$. If $y+T(C) \leq \lambda_{1}$, then

$$
|J|=T(C)-L(C) \geq T(B)-L(B) \geq|I| .
$$

If $y+T(C)>\lambda_{1}$, then by Lemma 3.2 and (3.7),

$$
\begin{aligned}
|J| & =\lambda_{1}-y-L(C) \geq L(B)-L(A)+T(C)-y-T(C)+T(B)-L(B) \\
& =T(B)-y-L(A)=|I| .
\end{aligned}
$$

Case 3: $y+L(A) \leq L(B) \leq y+T(A) \leq T(B)$.

By Lemma 3.2, $y+T(C) \leq \lambda_{1}$. If $y+L(C) \geq \mu_{1}$, then we argue as in Case 2 . If $y+L(C)<\mu_{1}$, then by Lemma 3.2 and $(3.7)$,

$$
\begin{aligned}
|J| & =y+T(C)-\mu_{1} \geq y+L(C)+T(B)-L(B)-T(B)+T(A)-L(C) \\
& =y+T(A)-L(B)=|I| .
\end{aligned}
$$

The proof is complete. 
The following theorem will be useful for the calculation of Denjoy indices.

Theorem 3.4 (Intersection Theorem). Let $x \in P$ with $x>1 / 2$. Let $(a, b)$, $(c, d)$ be two complementary intervals of $P$ with $0<a<b<1 / 2<x \leq c<$ $d<1$. Assume that $I:=(a, b) \cap(2 x-d, 2 x-c)$ is nonempty. Then there exists another complementary interval $\left(c_{1}, d_{1}\right)$ with $x \leq c_{1}<d_{1}<1$ such that the length of $\left(\mu_{1}, \lambda_{1}\right) \cap\left(2 x-d_{1}, 2 x-c_{1}\right)$ is at least $|I|$.

Proof. We use Lemma 3.3 with $y:=2 x-1>0$. Note that $(c, d)$ is a complementary interval of $P$ if and only if $(2 x-d, 2 x-c)$ is a complementary interval of $y+P$. Let $(a, b)=(L(B), T(B))$ and $(2 x-d, 2 x-c)=(y+$ $L(A), y+T(A))$. Since neither $A$ nor $B$ contain 1, condition (3.3) is satisfied. Let $C$ be defined as in (3.4). Set $\left(2 x-d_{1}, 2 x-c_{1}\right):=(y+L(C), y+T(C))$. Let $J$ be defined as in Lemma 3.3. By Lemma 3.3, $|J| \geq|I|$ which is the desired statement.

Of course, a similar theorem holds when $x<1 / 2$.

\section{Simplified Calculation of Denjoy Indices}

Consider a uniform symmetric perfect set $P=P(\eta)$. For $x \in P$ and $x<1 / 2$, we define $\gamma(x)$ as $\beta(x)$ but allowing only $(a, b)=(1-1 / \eta, 0)$, that is,

$$
\gamma(x):=\sup \left\{\begin{array}{l|l}
\frac{t}{s} & \begin{array}{c}
x \leq c<d, \text { and }(c, d) \text { is a } \\
\text { complementary interval of } \mathrm{P}
\end{array}
\end{array}\right\},
$$

where

$$
s=\max \{x, c-x\}, \quad t=\min \{x-1+1 / \eta, d-x\} .
$$

Similarly, for $x \in P$ and $x>1 / 2$, allowing only $(c, d)=(1,1 / \eta)$, define

$$
\gamma(x):=\sup \left\{\frac{t}{s} \mid \begin{array}{c}
a<b \leq x, \text { and }(a, b) \text { is a } \\
\text { complementary interval of } \mathrm{P}
\end{array}\right\},
$$

where

$$
s=\max \{x-b, 1-x\}, \quad t=\min \{x-a, 1 / \eta-x\} .
$$

Clearly, we have that $\gamma(x)=\gamma(1-x)$ and $\gamma(x) \leq \beta(x)$ for all $x \in P$.

Theorem 4.1. For every $x \in P=P(\eta)$,

$$
\beta(x)=\sup _{m \geq 0} \gamma\left(S^{m} x\right) \quad \text { and } \quad \alpha(x)=\limsup _{m \rightarrow \infty} \gamma\left(S^{m} x\right) .
$$


Proof. Let $x \in P$. By Lemma 2.2, we need only verify the equation for $\beta(x)$. By Lemma 2.1, $\gamma\left(S^{m} x\right) \leq \beta\left(S^{m} x\right) \leq \beta(x)$, hence $\beta(x) \geq \sup _{m \geq 0} \gamma\left(S^{m} x\right)$. To show the reversed inequality, let $(a, b),(c, d)$ be complementary intervals of $P$ with $a<b \leq x \leq c<d$. Let $s, t$ be defined by (1.1). The proof will be complete once we have shown that

$$
\frac{t}{s} \leq \gamma\left(S^{m} x\right) \text { for some } m \geq 0
$$

We may assume that $x<1 / 2$. If the level of $(a, b)$ is 0 , then (4.5) is true with $m=0$. If the level of $(c, d)$ is 0 , then $t / s<1$ unless $(a, b)$ has also level 0. Hence (4.5) is true. Now assume that the levels of $(a, b)$ and $(c, d)$ are at least 1 . Choose the maximal $n \geq 0$ such that $a$ and $d$ both belong to the same component $J$ of $P_{n}$ (recall that $P_{m}, m \in \mathbb{N}$, is the set that we obtain by removing all of the complementary intervals of level at most $m$ from $[0,1]$ and $\left.P_{0}:=[0,1]\right)$. Assume that $x$ lies in the left interval $H$ of $J \cap P_{n+1}$ (the other case is similar.) Because of the maximality of $n$, there are two possibilities: 1) $(c, d)$ has level $n+1$ and lies in the middle of $J$ or 2) $(c, d)$ lies in the right interval of $J \cap P_{n+1}$. Note that $S^{n}(P \cap J)$ is equal to $P$. Therefore, in the second case, by the Intersection Theorem, we may replace $(a, b)$ by another complementary interval $\left(a_{1}, b_{1}\right)$ and $(c, d)$ by the complementary interval $\left(c_{1}, d_{1}\right)$ in the middle of $J$ in such a way that the corresponding quotient $t_{1} / s_{1}$ is larger than $t / s$. Hence, in order to show (4.5), it is sufficient to consider the first case. If $x$ belongs to the left interval of $H \cap P_{n+2}$, then $t / s<1$. So assume that $x$ belongs to the right interval of $H \cap P_{n+2}$. Now the affine-linear extension of the restriction of $S^{n+1}$ to $H$ maps $H$ onto $[0,1], x$ to $S^{n+1} x,(a, b)$ to a complementary interval of $P$ and $(c, d)$ to $(1,1 / \eta)$. Hence (4.5) is true with $m=n+1$.

It is easy to see that, if $x<1 / 2$ and $S x<1 / 2$, then $\gamma(S x) \leq \gamma(x)$. The same is true if $x>1 / 2$ and $S x>1 / 2$. This means that some of the numbers $\gamma\left(S^{m} x\right)$ can be omitted in the formula for $\beta(x)$ in Theorem 4.1. For example, if $x$ is given by the sequence $000110 \ldots$, then $\gamma\left(S^{2} x\right) \leq \gamma(S x) \leq \gamma(x)$ and $\gamma\left(S^{4} x\right) \leq \gamma\left(S^{3} x\right)$; hence $\gamma(S x), \gamma\left(S^{2} x\right)$ and $\gamma\left(S^{4} x\right)$ cannot contribute to the supremum $\sup _{m \geq 0} \gamma\left(S^{m} x\right)$.

We note the following corollary of Theorem 4.1.

Corollary 4.2. Let $x$ be an $r$-periodic point of $P=P(\eta)$, that is, $S^{r} x=x$. Then

$$
\alpha(x)=\beta(x)=\max \left\{\gamma\left(S^{m} x\right) \mid m=0, \ldots, r-1\right\} .
$$

The supremum (4.1) defining $\gamma(x)$ is taken over an infinite set of $t / s$ quotients. We now show that finitely many of these quotients suffice. For 
each $k \in \mathbb{N}$, let $\tau_{k} \in(0,1)$ be the unique solution of

$$
\mu_{k}=\frac{1-\eta}{2-\eta}
$$

The solution is unique because $\mu_{k}$ is an increasing function of $\eta$ whereas the right-hand side of (4.6) is a decreasing function of $\eta$.

Lemma 4.3. Let $\ell \in \mathbb{N}, 0<\eta \leq \tau_{\ell}$ and $x \in P=P(\eta)$ with $x<1 / 2$. Then

$$
\gamma(x) \geq 1+\frac{\lambda_{\ell}-\mu_{\ell}}{x-1+1 / \eta} .
$$

Proof. We claim that there is a complementary interval $(c, d)$ of level at most $\ell$ to the right of $x$ whose intersection with the reflection of $(1-1 / \eta, 0)$ at $x$ has length at least $\lambda_{\ell}-\mu_{\ell}$. In fact, write $[0,1]$ as a disjoint union of the complementary intervals of $P$ of level between 1 and $\ell$ and a collection of closed intervals of length $\mu_{\ell}$.

The claim follows from the inequality $1 / \eta-1 \geq \mu_{\ell}+2\left(\lambda_{\ell}-\mu_{\ell}\right)$ which is true because it is equivalent to $\eta \leq \tau_{\ell}$. The desired inequality for $\gamma(x)$ follows easily.

Theorem 4.4. Let $\ell \in \mathbb{N}, 0<\eta \leq \tau_{\ell}$ and $x \in P=P(\eta)$ with $0<x<1 / 2$. Choose $q \geq \ell$ so large that

$$
\mu_{q-\ell+1}<\frac{x}{x-1+1 / \eta} .
$$

Then $\gamma(x)$ is equal to the supremum (4.1) taken only over the finitely many complementary intervals $(c, d)$ of $P$ whose level is at most $q$.

Proof. Let $(c, d)$ be a complementary interval of $P$ of level $m>q$ to the right of $x$. Let $s, t$ be defined by (4.2). Then

$$
\frac{t}{s} \leq \frac{x+\lambda_{m}-\mu_{m}}{x}=1+\frac{\lambda_{m}-\mu_{m}}{x}
$$

Since $m>q, \frac{\lambda_{m}-\mu_{m}}{x}<\frac{\lambda_{\ell}-\mu_{\ell}}{x-1+1 / \eta}$. Hence, by Lemma 4.3, $t / s<\gamma(x)$ which implies the statement of the theorem.

Corollary 4.2 and Theorem 4.4 make it possible to calculate Denjoy Indices of periodic points. The following computer generated table gives a periodic point that minimizes the Denjoy Index on $P(\eta)$ among all periodic points with period at most 11 for the indicated intervals for $\eta$. For example, the first line means that the point $x$ given by the two-periodic sequence $101010 \ldots$ has the minimal Denjoy Index among all periodic points with period at most 11 for $\eta$ between 0 and (approximately) 0.806963 . 


\begin{tabular}{|lcc|l|}
\hline 0 & $<\eta<$ & 0.806963 & 10 \\
\hline 0.806963 & $<\eta<$ & 0.874620 & 101 \\
\hline 0.874620 & $<\eta<$ & 0.945218 & 10 \\
\hline 0.945218 & $<\eta<$ & 0.957011 & 10101 \\
\hline 0.957011 & $<\eta<$ & 0.969684 & 1001 \\
\hline 0.969684 & $<\eta<$ & 0.985147 & 10 \\
\hline 0.985147 & $<\eta<$ & 0.991289 & 1010101 \\
\hline 0.991289 & $<\eta<$ & 0.992287 & 100101 \\
\hline 0.992287 & $<\eta<$ & 0.996159 & 10 \\
\hline 0.996159 & $<\eta<$ & 0.997104 & 101010101 \\
\hline 0.997104 & $<\eta<$ & 0.998058 & 1001 \\
\hline 0.998058 & $<\eta<$ & 0.999029 & 10 \\
\hline 0.999029 & $<\eta<$ & 0.999305 & 10101010101 \\
\hline 0.999305 & $<\eta<$ & 0.999513 & 10100101 \\
\hline
\end{tabular}

\section{The Denjoy Index of a Two-Periodic Point}

Let $P=P(\eta)$ for some $\eta \in(0,1)$. Let $w \in P$ be the point defined by the two-periodic sequence $101010 \ldots$. Then $w=\sum_{n=0}^{\infty} \lambda_{2 n+1}=\frac{1+\eta}{1+2 \eta}$. In the following we will obtain an explicit formula for $\alpha(w)$.

Since $S w=1-w$, by Corollary $4.2, \alpha(w)=\beta(w)=\gamma(w)$. Our goal is to show that only the complementary intervals $(a, b)=\left(a_{k}, b_{k}\right)$ of level $k \geq 0$ which are closest to $1-w$ are needed to evaluate $\gamma(w)$ according to (4.3). If $k$ is even, then a computation gives

$$
a_{k}=\frac{\eta}{1+2 \eta}\left(1-\frac{1+\eta-\eta^{2}}{\eta^{2}} \mu_{k}\right) \quad \text { and } \quad b_{k}=\frac{\eta}{1+2 \eta}\left(1-\mu_{k}\right) \text {. }
$$

If $k$ is odd, then we obtain

$$
a_{k}=\frac{\eta}{1+2 \eta}\left(1+\mu_{k}\right) \quad \text { and } \quad b_{k}=\frac{\eta}{1+2 \eta}\left(1+\frac{1+\eta-\eta^{2}}{\eta^{2}} \mu_{k}\right) .
$$

Note that

$$
a_{0}<b_{0}<a_{2}<b_{2}<a_{4}<b_{4}<\cdots<1-w<\cdots<a_{3}<b_{3}<a_{1}<b_{1} .
$$

For every even $k \geq 0$, let $\eta_{k} \in[0,1)$ be the unique solution of the equation

$$
\mu_{k}=\frac{1-\eta^{2}}{1+\eta-\eta^{2}}
$$


For odd $k$, let $\eta_{k} \in(0,1)$ be the unique solution of the equation $\mu_{k}=\frac{1-\eta}{\eta}$. Note that $0=\eta_{0}<\eta_{1}<\eta_{2}<\eta_{3}<\ldots$ and $\eta_{k} \rightarrow 1$. For example, we find that

$$
\eta_{1}=1 / \sqrt{2}=0.707106 \ldots, \quad \eta_{2}=0.871156 \ldots, \quad \text { and } \eta_{3}=0.903408 \ldots .
$$

Lemma 5.1. Let $k \geq 0$ be even. If $\eta_{k}<\eta \leq \eta_{k+1}$, then

$$
\begin{aligned}
2 w-b_{k+1}<1 & \leq 2 w-a_{k+1}<2 w-b_{k+2}<2 w-a_{k+2}<2 w-b_{k} \\
& \leq 1 / \eta<2 w-a_{k} .
\end{aligned}
$$

If $\eta_{k+1}<\eta \leq \eta_{k+2}$, then $2 w-a_{k+1}<1<2 w-b_{k+2}<2 w-a_{k+2}<1 / \eta<$ $2 w-b_{k}$.

Proof. It is easy to verify that the three inequalities $2 w-b_{k+1}<1,1 / \eta<$ $2 w-a_{k}$ and $\eta_{k}<\eta$ are equivalent. Also the inequalities $1 \leq 2 w-a_{k+1}$, $2 w-b_{k} \leq 1 / \eta$ and $\eta \leq \eta_{k+1}$ are equivalent. Finally, $\left(2 w-b_{k+2}, 2 w-a_{k+2}\right) \subset$ $(1,1 / \eta)$ for all $\eta \in\left(\eta_{k}, \eta_{k+2}\right]$.

Let $s=s_{k}, t=t_{k}$ be given by (4.4) with $a=a_{k}, b=b_{k}$ and $x=w$. By the lemma, if $k \geq 0$ is even and $\eta_{k}<\eta \leq \eta_{k+2}$, then

$$
\frac{t_{k+2}}{s_{k+2}}=\frac{w-a_{k+2}}{w-b_{k+2}}=\frac{\eta+\left(1+\eta-\eta^{2}\right) \mu_{k+2}}{\eta\left(1+\eta \mu_{k+2}\right)} .
$$

If $\eta_{k}<\eta \leq \eta_{k+1}$, then

$$
\frac{t_{k+1}}{s_{k+1}}=\frac{w-a_{k+1}}{1-w}=\frac{1-\eta \mu_{k+1}}{\eta} \text { and } \frac{t_{k}}{s_{k}}=\frac{1 / \eta-w}{w-b_{k}}=\frac{1+\eta-\eta^{2}}{\eta\left(1+\eta \mu_{k}\right)} .
$$

Theorem 5.2. Let $k \geq 0$ be even. If $\eta_{k}<\eta \leq \eta_{k+1}$, then

$$
\alpha(w)=\max \left\{\frac{t_{k}}{s_{k}}, \frac{t_{k+2}}{s_{k+2}}\right\} .
$$

If $\eta_{k+1}<\eta \leq \eta_{k+2}$, then $\alpha(w)=\frac{t_{k+2}}{s_{k+2}}$.

Proof. Assume that $\eta_{k}<\eta \leq \eta_{k+2}$. We claim that all the complementary intervals $(a, b)$ of level at least $k+3$ are without interest in the formula (4.3) for $\gamma(w)$. Let $(a, b)$ be such an interval and let $s, t$ be as in (4.4) with $x=w$. Then

$$
\frac{t}{s} \leq 1+\frac{\lambda_{k+3}-\mu_{k+3}}{1-w}
$$


Since $\left(2 w-b_{k+2}, 2 w-a_{k+2}\right) \subset(1,1 / \eta)$,

$$
\frac{t_{k+2}}{s_{k+2}} \geq \frac{1 / \eta-w}{1 / \eta-w-\lambda_{k+2}+\mu_{k+2}}>1+\frac{\lambda_{k+2}-\mu_{k+2}}{1 / \eta-w} .
$$

Hence, we are done once we have shown that $\frac{\lambda_{k+3}-\mu_{k+3}}{1-w} \leq \frac{\lambda_{k+2}-\mu_{k+2}}{1 / \eta-w}$ which is equivalent to $1 \leq 2 \eta^{2}$. The latter is true when $\eta \geq \eta_{1}=1 / \sqrt{2}$. If $k=0$ and $0<\eta \leq \eta_{1}$, then one can show by a more careful estimation that the righthand side of the inequality (5.2) is less than $t_{2} / s_{2}$ (without using an estimate for $\left.t_{2} / s_{2}\right)$. This establishes our claim.

Between $\left(a_{k}, b_{k}\right)$ and $\left(a_{k+1}, b_{k+1}\right)$ there are no complementary intervals of level at most $k+1$, and exactly one of level $k+2$, namely $\left(a_{k+2}, b_{k+2}\right)$. By Lemma $5.1,2 w-b_{k+1}<1$ and $2 w-a_{k}>1 / \eta$. Thus the only complementary intervals $(a, b)$ of level at most $k+2$ which are of interest in (4.3) are $\left(a_{m}, b_{m}\right)$ with $m=k, k+1, k+2$.

We have shown that $\alpha(w)$ is the maximum of the three quotients $t_{m} / s_{m}$ with $m=k, k+1, k+2$. If $\eta_{k}<\eta \leq \eta_{k+1}$, then $t_{k+1} / s_{k+1} \leq t_{k} / s_{k}$ because a calculation shows that the latter is equivalent to $\eta \leq \eta_{k+1}$. Therefore, the statement of the theorem is proved in this case. If $\eta_{k+1}<\eta \leq \eta_{k+2}$, then $t_{k} / s_{k}$ and $t_{k+1} / s_{k+1}$ are both at most 1 , hence the theorem is proved in this case as well.

By some additional work, one can show that, if $k \geq 0$ is even, there is a uniquely determined $\nu_{k} \in\left(\eta_{k}, \eta_{k+1}\right)$ such that $\alpha(w)=t_{k} / s_{k}$ for $\eta_{k}<\eta \leq \nu_{k}$ and $\alpha(w)=t_{k+2} / s_{k+2}$ for $\nu_{k}<\eta \leq \eta_{k+2}$. For example,

$$
\alpha(w)=\frac{t_{0}}{s_{0}}=\frac{1+\eta-\eta^{2}}{\eta(1+\eta)} \quad \text { if } 0<\eta \leq \nu_{0}=0.637708 \ldots
$$

and

$$
\alpha(w)=\frac{t_{2}}{s_{2}}=\frac{1+3 \eta+2 \eta^{2}-\eta^{3}}{1+2 \eta+\eta^{2}+\eta^{3}} \quad \text { if } \nu_{0}<\eta \leq \eta_{2} .
$$

We now turn to the question whether $\alpha(w)$ is the minimal Denjoy Index of points in $P(\eta)$. Note that the solutions $\eta=\tau_{k}$ defined by (4.6) satisfy

$$
0=\eta_{0}<\tau_{1}<\eta_{1}<\tau_{2}<\eta_{2}<\tau_{3}<\ldots
$$

Theorem 5.3. Let $k \geq 2$ be even and let $\nu_{k} \leq \eta \leq \tau_{k+2}$. Then $\alpha(w)=$ $\min \alpha(P)$. 
Proof. By definition of $\nu_{k}$ and Theorem 5.2, we have that $\alpha(w)=t_{k+2} / s_{k+2}$. Since $\left(2 w-b_{k+2}, 2 w-a_{k+2}\right) \subset(3 w-1,1 / \eta)$, we find that

$$
\alpha(w)=\beta(w) \leq \frac{2 w-1+\lambda_{k+2}-\mu_{k+2}}{2 w-1}=1+\frac{\lambda_{k+2}-\mu_{k+2}}{2 w-1} .
$$

By Theorem 2.3, to prove the theorem it is sufficient to show that $\beta(y)>\beta(w)$ for every $y \in P$ different from $w$ and $1-w$. Let $y \in P$ be different from $w$ and $1-w$. Then its representing 01-sequence contains two consecutive 1's or 0 's. By Lemma 2.1 and since $\beta(y)=\beta(1-y)$ it is sufficient to consider the case that the sequence starts with 00 . Then $0 \leq y \leq \mu_{2}$. Therefore, Lemma 4.3 yields $\beta(y) \geq \gamma(y) \geq 1+\frac{\lambda_{k+2}-\mu_{k+2}}{\mu_{2}-1+1 / \eta}$. Using (5.4), $\beta(y)>\beta(w)$ will follow if $\frac{1}{2 w-1}<\frac{1}{\mu_{2}+1 / \eta-1}$. This inequality is equivalent to $\eta>\eta_{2}$ which is true.

It is shown in [4] that $\alpha(w)=\min \alpha(P)$ for all $0<\eta \leq(\sqrt{5}-1) / 2=$ $0.618033 \ldots$. By a more careful estimation of $\beta_{2}(x)$ for $0 \leq x \leq \mu_{2}$, one can show that this remains true for all $0<\eta \leq \tau_{2}=0.801937 \ldots$ in agreement with our table in Section 4. The table also shows that $w$ does not minimize $\alpha$ for all $\eta$. For example, if $y \in P$ is given by the 3 -periodic sequence (101)(101) $\ldots$ and $\eta=0.85$, then (5.3) gives $\alpha(w)=1.085282 \ldots$.

Using the results from Section 4 one can compute $\alpha(y)=1.078163 \ldots$ which is smaller.

\section{References}

[1] P. S. Bullen, Denjoy's Index and Porosity, Real Analysis Exchange, 10 (1984-5), 85-144.

[2] A. Denjoy, Leçons sur le Calcul des Coefficients d'Une Série Trigonométrique I-IV, Paris, 1941-1949.

[3] M. J. Evans, P. D. Humke and K. Saxe, A Symmetric Porosity Conjecture of Zajiček, Real Analysis Exchange, 17 (1991-2), 258-271.

[4] R. Svetic and H. Volkmer, The Denjoy Index and symmetric perfect sets, Italian Journal of Pure and Applied Mathematics, to appear. 